\title{
Viscoelastic properties of extracellular polymeric substances can strongly affect their washing efficiency from reverse osmosis membranes
}

\section{Supplementary information}

\author{
Diana Ferrando, Ali Nejidat, and Moshe Herzberg* \\ The Jacob Blaustein Institutes for Desert Research, Zuckerberg Institute for Water \\ Research, The Albert Katz International School of Desert Studies, Ben Gurion University \\ of the Negev, Sede Boqer Campus, Midreshet Ben Gurion 84990, Israel. \\ * Corresponding author phone: 972-8-6563520 e-mail: herzberg@ bgu.ac.il
}


Figure S1. Chemical structure of NOMEX polyamide material used for coating of QCM-D sensors.<smiles>CNc1cccc(NC(=O)c2cccc(C(C)(C)C)c2)c1</smiles>

Figure S2. Plate and frame RO unit closed loop system for washing EPS from the fouled RO membrane and analyzing permeate flux recovery.

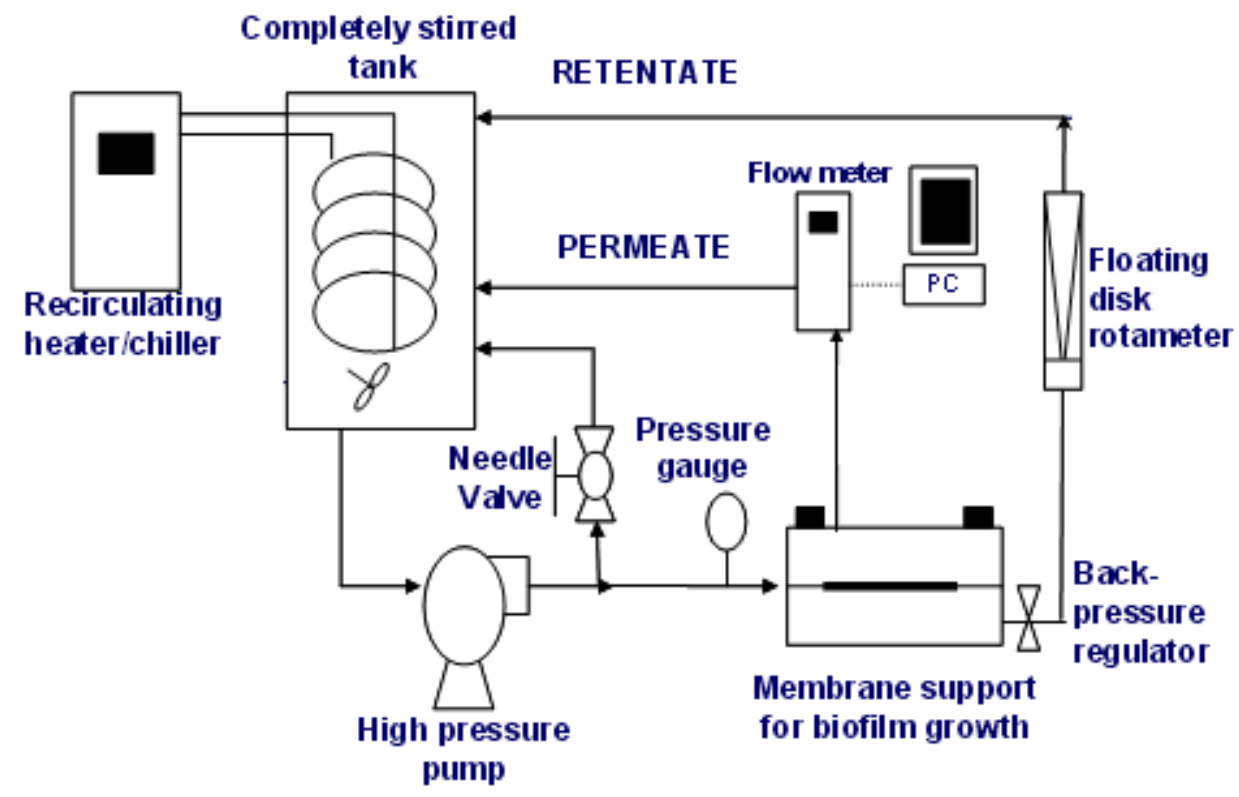


DLS Analysis. The distributions of diffusion coefficients for different EPS solutions extracted from the $P$. aeruginosa wild type, $\Delta p s l$ mutant and Psl overexpression mutant strains were measured using dynamic light-scattering (CGS-3, ALV, Langen, Germany). Duplicates of the EPS samples were dissolved in $50 \mathrm{mM} \mathrm{NaCl}$ background solution to a final concentration of $32.5 \mathrm{mg} \cdot \mathrm{L}^{-1}$ as TOC. All samples were filtered in $0.22 \mu \mathrm{m}$ hydrophilic PVDF filter (Millipore) prior to the measurement and $1 \mathrm{~mL}$ samples were placed in glass vials (Sigma-Aldrich, ISRAEL). The laser power was $20 \mathrm{~mW}$ at the HeNe laser line $(632.8 \mathrm{~nm})$. Correlograms were calculated by ALV/LSE 5003 correlator, which were collected at $90^{\circ}$, during $10 \mathrm{~s}$ for 20 times, at $25^{\circ} \mathrm{C}$. The correlograms were fitted with version of the program CONTIN to calculate the decay time. Diffusion coefficients were calculated from the decay time distribution.

Figure S3. Normalized scattering intensity distribution of the diffusion coefficients for the three EPS solutions extracted from $P$. aeruginosa wild type, $\Delta p s l$ mutant and $\Delta p s l /$ $P_{B A D}$-psl (Psl overexpression) strains suspended in $50 \mathrm{mM} \mathrm{NaCl}$.

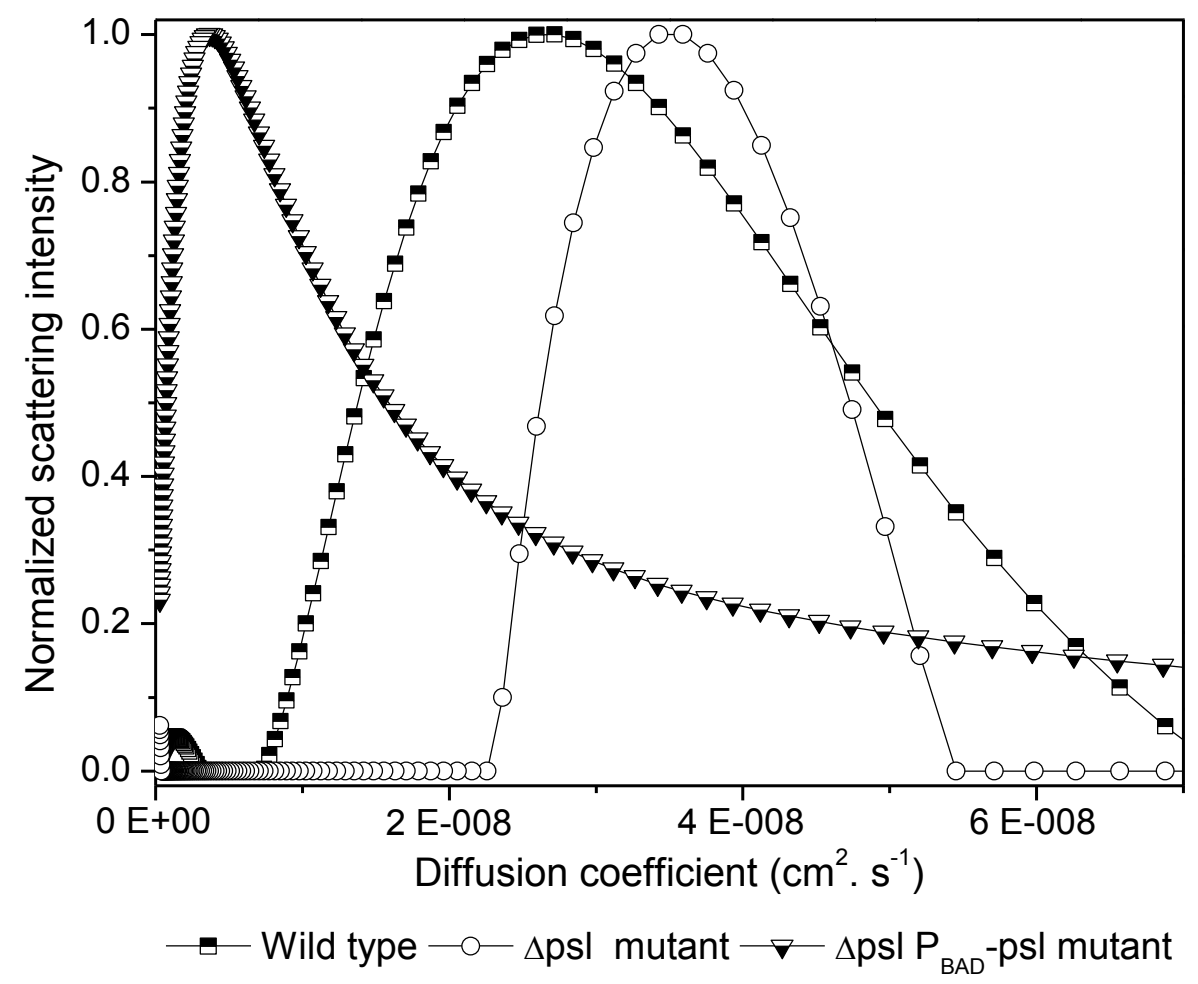


An explanation for reduced flux recovery at high shear rate conditions would be a selection for removal of relatively big particles or aggregates $(>1 \mu \mathrm{m})$ as shown in other studies, where removal of fouling layers was facilitated by inertial lift mechanism, while selecting for small particles to retain on the membrane $e^{1-4}$. In order to justify this explanation, DLS analysis of the three different types of EPS suspended in $50 \mathrm{mM} \mathrm{NaCl}$ solution was performed. Indeed, the distributions of the diffusion coefficients of the EPS suspensions (Figure S3) imply that the EPS from the $\Delta p s l$ mutant had smallest size distribution (deduced from the highest values of the diffusion coefficients). Smaller diffusion coefficients were analyzed for the EPS of the wild type followed by the smallest diffusion coefficient of the EPS, overexpressed with Psl (Figure S3). The associated sizes of the EPS imply that removal of big particles/aggregates from the EPS fouling layers, followed by compaction of the layers with smaller molecules that retain, can further reduce permeate flux. Those layers with smaller macromolecules and particles will likely acquire smaller porosity that consequently will elevate the transmembrane osmotic pressure $^{5,6}$. In the case of the EPS from wild type strain, elevation of the inertial lift at higher shear, will likely remove big EPS colloids and the retained smaller macromolecules will form a more compacted fouling layer. In the case of EPS overexpressed with Psl, the portion of small EPS colloids is relatively small, which probably could not support such elevation of fouling layer compaction. Further fundamental research on this exciting phenomenon is currently under investigation. 
Figure S4. Frequency (A) and dissipation (B) shifts measured for the polyamide (NOMEX) coated QCM-D sensors during adherence experiments of EPS (bulk concentration of $20 \mathrm{mg} \mathrm{l}^{-1}$ DOC) extracted from $P$. aeruginosa PAO1, $\Delta p s l$ mutant and $\Delta p s l / P_{B A D}$-psl mutant (Psl overexpression). The experiment included the following solution injection steps of 30 min each: (1) Baseline with DI water; (2) $\mathrm{NaCl}$ background solution; (3) EPS dissolved in background solution; (4) $\mathrm{NaCl}$ background solution; (5) DI water.

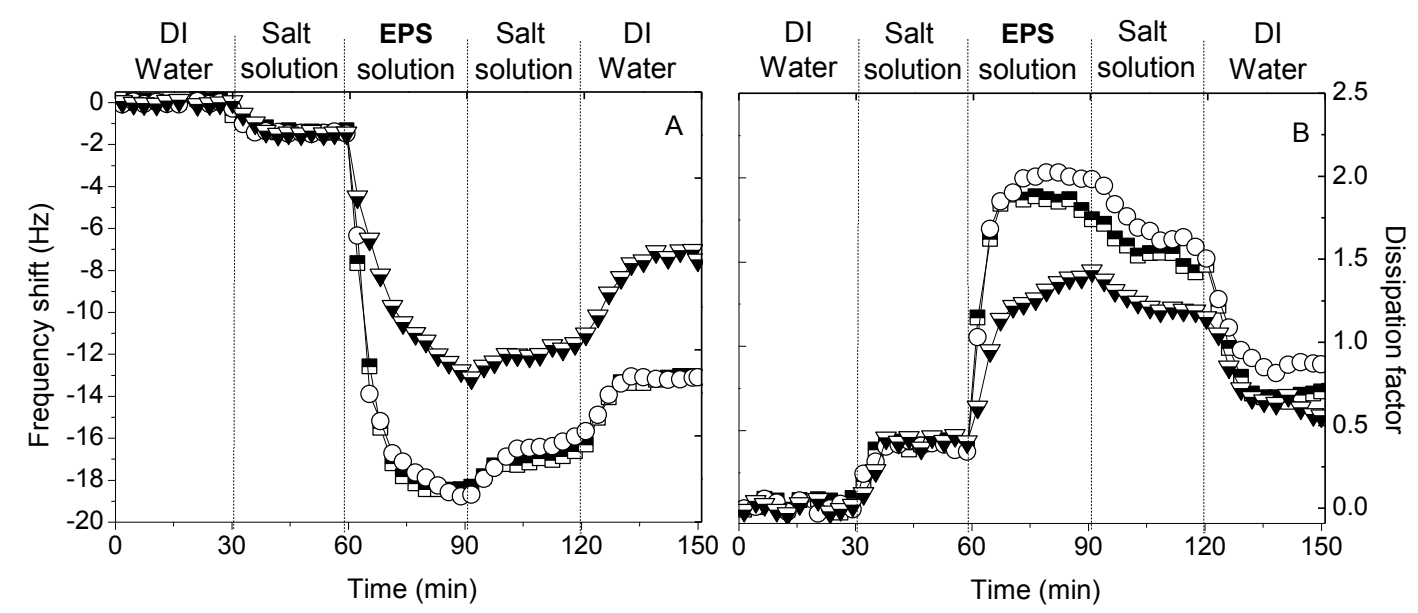


Figure S5. Frequency (A) and dissipation (B) shifts measured for the gold-coated QCMD sensors during adherence experiments of EPS (bulk concentration of $20 \mathrm{mg} \mathrm{l}^{-1}$ DOC) extracted from $P$. aeruginosa PAO1, $\Delta p s l$ mutant and $\Delta p s l / P_{B A D}-p s l$ mutant (Psl overexpression). The experiment included the following solution injection steps of 30 min each: (1) Baseline with DI water; (2) NaCl background solution; (3) EPS dissolved in background solution; (4) $\mathrm{NaCl}$ background solution; (5) DI water.

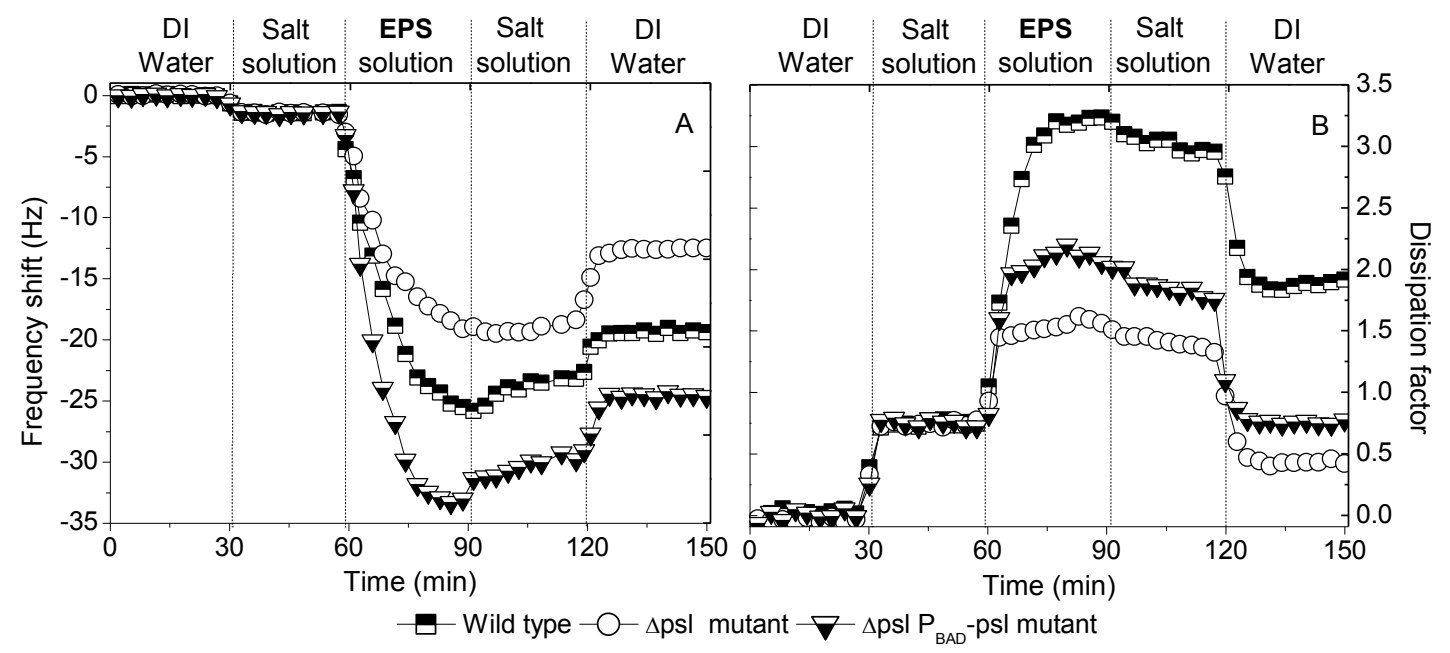

The results obtained from the experiments carried out on gold-coated sensors (Figure S5) revealed a different behavior compared to the experiments carried out on polyamide (NOMEX) (Figure S4). The EPS from the $\Delta p s l$ mutant showed the lowest adsorption rate followed by the EPS from wild type. Contrary to the previous experiments, the EPS extracted from the Pel overexpression strain showed the highest adherence to the goldcoated sensors. Accordingly to the EPS Zeta potential results, the positively charged gold surface of the sensor attracted more EPS originated from the Psl overexpression strain ($28.8 \mathrm{mV} \pm 1.25)$ followed by the wild type strain $(-21.3 \mathrm{mM} \pm 1.49)$ and the lowest adherence was observed for the $\Delta p s l$ mutant $(-15.8 \mathrm{mV} \pm 0.44)$. 


\section{References}

1. Zydney, A. L.; Colton, C. K., A concentration polarization model for the filtrate flux in cross-flow microfiltration of particulate suspensions. In Chemical engineering communications, 1986; Vol. 47, pp 1-21.

2. Davis, R. H., Modeling of fouling of crossflow microfiltration membranes. In Separation and purification methods, 1992; Vol. 21, pp 75-126.

3. Baker, R. J.; Fane, A. G.; Fell, C. J. D.; Yoo, B. H., Factors affecting flux in crossflow filtration. In Desalination, 1985; Vol. 53, pp 81-93.

4. Belfort, G.; Davis, R. H.; Zydney, A. L., The behavior of suspensions and macromolecular solutions in cross-flow microfiltration. Journal of Membrane Science 1994, 96, (1-2), 1-58.

5. Hoek, E. M. V.; Kim, A. S.; Elimelech, M., Influence of crossflow membrane filter geometry and shear rate on colloidal fouling in reverse osmosis and nanofiltration separations. Environmental Engineering Science 2002, 19, (6), 357-372.

6. Hoek, E. M. V.; Elimelech, M., Cake-enhanced concentration polarization: A new fouling mechanism for salt-rejecting membranes. Environmental Science \& Technology 2003, 37, (24), 5581-5588. 